\title{
Light from Schwarzschild black holes in de Sitter expanding universe
}

\author{
Ion I. Cotăescu ${ }^{1, \mathrm{a}}$ (1) \\ ${ }^{1}$ West University of Timişoara, V. Pârvan Ave. 4, 300223 Timisoara, Romania
}

Received: 5 November 2020 / Accepted: 29 December 2020 / Published online: 16 January 2021

(C) The Author(s) 2021

\begin{abstract}
A new method is applied for deriving simultaneously the redshift and shadow of a Schwarzschild black hole moving freely in the de Sitter expanding universe as recorded by a remote co-moving observer. This method is mainly algebraic, focusing on the transformation of the conserved quantities under the de Sitter isometry relating the black hole co-moving frame to observer's one. Hereby one extracts the general expressions of the redshifts and shadows of the black holes having peculiar velocities but their expressions are too extended to be written down here. Therefore, only some particular cases and intuitive expansions are presented while the complete results are given in an algebraic code (Cotăescu in Maple code BH01, https://physics.uvt.ro/ $\sim \operatorname{cota} /$ CCFT/codes, 2020).
\end{abstract}

\section{Introduction}

The light emitted by cosmic objects is one of the principal sources of empirical data in astrophysics. An important accessible observable is the redshift which encapsulates information about the cosmic expansion and possible peculiar velocity of the observed object. For separating these two contributions one combined so far the Lemaittre rule $[2,3]$ of Hubble's law [4], governing the cosmological effect, [5-7] with the usual theory of the Doppler effect of special relativity [8] even though there is evidence that our universe is expanding.

Recently, we proposed an improvement of this approach replacing special relativity with our de Sitter relativity $[9,10]$ where local charts of the same type, playing the role of inertial frames, are related among themselves through de Sitter isometries. In the case of the longitudinal Doppler effect, when a point-like source is moving along the axis observersource, we obtained a redshift formula having a new term

\footnotetext{
a e-mail: i.cotaescu@e-uvt.ro (corresponding author)
}

combining the cosmological and kinetic contributions in a non-trivial manner [11].

The next step is to extend this method to the black holes which, in general, are no point-like sources. The light emitted by a black hole comes from an apparent source situated on a sphere, surrounding the black hole, which is observed as the black hole shadow. When this is not negligible, as in the case of the object M87 [12,13], and the black hole may have a peculiar velocity, the Doppler effect is no longer longitudinal such that the transverse contributions due to the black hole shadow must be evaluated. This can be done only by studying simultaneously the Doppler effect and black hole shadow in the same theoretical framework.

The black hole shadow was extensively studied by many authors with the help of a geometric method which exploits the tangent to the null geodesic in the point where this meets the observer. Thus the shadows of the Schwarzschild black holes were studied in Minkowski space-time [14] or in expanding universes [15-20], while for the rotating black holes the Kerr [21,22] or Kerr-de Sitter [23-26] metrics were considered. Other recent studies focused on more complex models which were studied with the same geometric method [27-43] but which is not suitable for studying the Doppler effect.

As in this paper we would like to study the relation between the redshifts and shadows of the Schwarzschild black holes having peculiar velocities in de Sitter expanding universe we must abandon the geometric method adopting the algebraic approach of Ref. [11] which is suitable for deriving the redshift. This is based on the de Sitter relativity where we may relate the moving black hole proper frames to those of remote co-moving observers freely falling in the de Sitter expanding universe.

We start supposing that our expanding universe is satisfactory described by the expanding portion of a $(1+3)$ dimensional de Sitter manifold. As the actual observations show with reasonable accuracy that this universe is spatially 
flat, we consider only local charts with Painlevé coordinates [44] since these have flat space sections. These local charts are de Sitter co-moving frames [45] where the coordinates are the cosmic time and Cartesian or spherical space coordinates. These frames may carry observers related among themselves through the de Sitter isometries which transform simultaneously the coordinates and the conserved quantities $[9,10]$.

The Schwarzschild-de Sitter black holes are usually considered in proper frames with static coordinates and Kottler metric [46]. However, here we prefer the corresponding comoving frames with Painlevé coordinates whose metrics have the same asymptotic behavior as the metric of the observer co-moving frame. Then for a remote observer the black hole co-moving frame appears as an empty de Sitter one which can be related to observer's co-moving frame through a de Sitter isometry, in accordance with the relative motion of the black hole with respect to observer.

We assume that at the initial moment when the black hole emits the photon this is translated and has a relative velocity with respect to the remote observer known as peculiar velocity. For avoiding extremely complicated calculations we assume that this velocity is longitudinal, in the black holeobserver direction. Thus we start with precise initial conditions determining the suitable isometry relating the black hole and observer proper frames. This will give the conserved quantities measured in the observer's frame we need for extracting physical results without resorting to geodesics or other geometric objects. For this reason we say that our method is algebraic, observing that there are some advantages among them the principal one is of a coherent framework offered by the de Sitter relativity which prevent us of using supplemental hypotheses.

In this approach we derive the related redshift and shadow of a Schwarzschild black hole freely moving in the de Sitter expanding universe. Our principal new results are a closed formula of the black hole shadow depending on its peculiar velocity and the corrections to our new redshift formula derived in Ref. [11] due to the dimension of the black hole shadow. These corrections are too complicated to be written down here but can be derived with the help of an algebraic code on computer [1].

We start in the second section with a brief review of the metrics of the black hole and observer co-moving frames with Cartesian or spherical coordinates, revisiting the equation giving the geodesic shapes in the black hole co-moving frames. In the next section we present the solutions of this equation representing the null geodesics around the black hole. These are the circular geodesics on the photon sphere and the Darwin $[47,48]$ spiral geodesics which determine the black hole shadow. The fourth section is devoted to the de Sitter isometries relating the conserved quantities measured in different co-moving frames. In the next section we obtain our new results assuming that a remote observer sees that the light is emitted by an apparent source on a null de Sitter geodesic whose conserved quantities can be determined. Furthermore, by using an isometry formed by a translation followed by a Lorentzian isometry we obtain the conserved quantities in the observer's co-moving frame from which we extract the observed redshift and shadow of the moving black hole. More specific, the redshift results from the observed energy while the angular radius of the black hole shadow is derived by using the components of the photon momentum in the observer's origin, where the photon angular momentum must vanish. These results are elementary but with a large number of terms that cannot be written here in the general case of a moving black hole. Consequently, we restrict ourselves to presenting here only their series expansions with respect to a common small parameter, the particular case when the relative velocity vanishes and the flat limit. As mentioned, the complete results which cannot be written here are given in an algebraic code on computer [1]. In order to convince oneself that the flat limit is correct we derive in Appendix B the results that can be obtained by applying our algebraic method to a Schwarzschild black hole in Minkowski flat space-time. Finally we present some concluding remarks.

As our approach may be applied even in quantum theory we introduce a special notation denoting by $\omega_{\mathrm{H}}=\sqrt{\frac{\Lambda}{3}} c$ the de Sitter Hubble constant (frequency) since $H$ is reserved for the Hamiltonian operator [49]. Moreover, the Hubble time $t_{\mathrm{H}}=\frac{1}{\omega_{\mathrm{H}}}$ and the Hubble length $l_{\mathrm{H}}=\frac{c}{\omega_{\mathrm{H}}}$ will have the same form in the natural Planck units with $c=\hbar=G=1$ we use here.

\section{Co-moving frames}

The frames of the spherically symmetric static systems in a $(1+3)$-dimensional isotropic pseudo-Riemannian manifold, $(M, g)$, are static local charts $\{x\}$ with spherical symmetry whose coordinates $x^{\mu}(\alpha, \mu, v, \ldots=0,1,2,3)$ can be chosen in different manners. In what follows we consider either Cartesian space coordinates $\mathbf{x}=\left(x^{1}, x^{2}, x^{3}\right)$ or associated spherical ones $(r, \theta, \phi)$ with $r=|\mathbf{x}|$. The traditional static frames, $\left\{t_{s}, \mathbf{x}\right\}$ or $\left\{t_{s}, r, \theta, \phi\right\}$, depend on the static time $t_{s}$ having the line elements

$$
\begin{aligned}
\mathrm{d} s^{2} & =f(|\mathbf{x}|) \mathrm{d} t_{s}^{2}+\left(\frac{\mathbf{x} \cdot \mathrm{d} \mathbf{x}}{|\mathbf{x}|}\right)^{2}\left(1-\frac{1}{f(|\mathbf{x}|)}\right)-\mathrm{d} \mathbf{x} \cdot \mathrm{d} \mathbf{x} \\
& =f(r) \mathrm{d} t_{s}^{2}-\frac{\mathrm{d} r^{2}}{f(r)}-r^{2} \mathrm{~d} \Omega^{2}
\end{aligned}
$$

where $\mathrm{d} \Omega^{2}=\mathrm{d} \theta^{2}+\sin ^{2} \theta \mathrm{d} \phi^{2}$. These line elements can be put at any time in Painlevé forms [44], 


$$
\begin{aligned}
\mathrm{d} s^{2} & =f(|\mathbf{x}|) \mathrm{d} t^{2}+\frac{2}{|\mathbf{x}|} \sqrt{1-f(|\mathbf{x}|)} \mathbf{x} \cdot \mathrm{d} \mathbf{x} \mathrm{d} t-\mathrm{d} \mathbf{x} \cdot \mathrm{d} \mathbf{x} \\
& =f(r) \mathrm{d} t^{2}+2 \sqrt{1-f(r)} \mathrm{d} t \mathrm{~d} r-\mathrm{d} r^{2}-r^{2} \mathrm{~d} \Omega^{2}
\end{aligned}
$$

substituting in Eqs. (1) and (2)

$t_{s}=t+\int \mathrm{d} r \frac{\sqrt{1-f(r)}}{f(r)}$,

where $t$ represents the cosmic time of the frames having flat space sections with Cartesian, $\{t, \mathbf{x}\}$, or spherical, $\{t, r, \theta, \phi\}$, coordinates.

Here we focus on a Schwarzschild black hole of mass $M$ embedded in the de Sitter expanding universe for which the metric (2) of its static frame has the Kottler [46] (or Schwarzschild-de Sitter) form with

$f(r)=1-\frac{2 M}{r}-\omega_{\mathrm{H}}^{2} r^{2}$.

where, as mentioned before, $\omega_{\mathrm{H}}$ is the de Sitter Hubble constant in our notation. The corresponding frames with Painlevé coordinates have the asymptotic behavior of the de Sitter comoving frames with $f(r) \rightarrow f_{0}(r)=1-\omega_{\mathrm{H}}^{2} r^{2}$. For this reason we say that the black hole frames with Painlevé coordinates, denoted by $\{t, \mathbf{x}\}_{\mathrm{BH}}$ and $\{t, r, \theta, \phi\}_{\mathrm{BH}}$, are the comoving frames of the Schwarzschild black hole in de Sitter expanding universe.

The frames of the remote observers, $\{t, \mathbf{x}\}$ and $\{t, r, \theta, \phi\}$, located in the asymptotic zone, are genuine de Sitter comoving frames where the astronomical observations are performed and recorded. The observers stay at rest in the origins of their own frames evolving along the unique time-like Killing vector field of the de Sitter geometry which is not time-like everywhere but has this property just in the null cone where the observations are allowed [49].

Here we use simultaneously Cartesian and spherical coordinates since the Cartesian coordinates are suitable for studying the conserved quantities and the transformation rules under isometries while the spherical coordinates help one to integrate the geodesic equations. For example, the spherical symmetry is obvious in Cartesian coordinates where the metrics (1) and (3) are invariant under the global rotations $x^{i} \rightarrow R_{j}^{i} x^{j}$ such that we can use the vector notation. On the other hand, only in spherical coordinates one can integrate the geodesics equations in the black hole co-moving frame we revisit briefly in the next.

In the frame $\{t, r, \theta, \phi\}_{\mathrm{BH}}$ with the line element (4) the conserved quantities along geodesics are the energy $E$ and angular momentum $\mathbf{L}$. These give rise to the prime integrals of a geodesic of a particle of mass $m$ moving in the equatorial plane of the black hole (with fixed $\theta=\frac{\pi}{2}$ ) as

$E=f(r) \dot{t}+\sqrt{1-f(r)} \dot{r}$,
$L=r^{2} \dot{\phi}$

where 'dot' denotes the derivatives with respect to the affine parameter $\lambda$ which satisfies $\mathrm{d} s=m \mathrm{~d} \lambda$. The third prime integral comes from the line element in the equatorial plane, which reads

$f(r) \dot{t}^{2}+2 \sqrt{1-f(r)} \dot{t} \dot{r}-\dot{r}^{2}-r^{2} \dot{\phi}^{2}=m^{2}$.

as it results from Eq. (4). Hereby one may derive the function $r(\phi)$ substituting

$r \rightarrow r(\phi), \quad \dot{r} \rightarrow \frac{\mathrm{d} r(\phi)}{\mathrm{d} \phi} \dot{\phi}=\frac{L}{r(\phi)^{2}} \frac{\mathrm{d} r(\phi)}{\mathrm{d} \phi}$

After a little calculation, combining the above prime integrals, one obtains the well-known equation

$$
\left(\frac{\mathrm{d} r(\phi)}{\mathrm{d} \phi}\right)^{2}-r(\phi)^{4} \frac{E^{2}}{L^{2}}+r(\phi)^{2} f[r(\phi)]\left(1+r(\phi)^{2} \frac{m^{2}}{L^{2}}\right)=0
$$

giving the geodesic shapes but which is not enough for finding the time behavior of the functions $\phi(t)$ and $r(t)=r[\phi(t)]$ for which one must apply special methods [50].

Note that Eq. (11) derived in the co-moving frame is the same as that of the static frame since this equation is static giving only the shape of trajectory in the same space coordinates. In fact, the time evolution on geodesics is quite different in the static and co-moving frames.

\section{Light around black holes}

The problem of the gravitational lensing which has a long history [51] was studied in general relativity first by Einstein and Eddington [52] but was solved by Darwin [47,48] which derived the null geodesics around the photon sphere of a Schwarzschild black hole in the flat Minkowski space-time. Applying the same commonly used method [53-58] we may inspect briefly the null geodesics in the co-moving frame $\{t, r, \theta, \phi\}_{B H}$ of the Schwarzschild-de Sitter system.

The shapes of the photon geodesics are given by the functions $r(\phi)$ which satisfy Eq. (11) with $m=0$ that now reads

$$
\left(\frac{\mathrm{d} r(\phi)}{\mathrm{d} \phi}\right)^{2}-r(\phi)^{4} \frac{E_{p h}^{2}}{L_{p h}^{2}}+r(\phi)^{2} f[r(\phi)]=0
$$

This equation has two types of solutions, namely circular geodesics on the photon sphere and associated spiral geodesics [47]. The circular geodesics satisfy simultaneously the conditions 

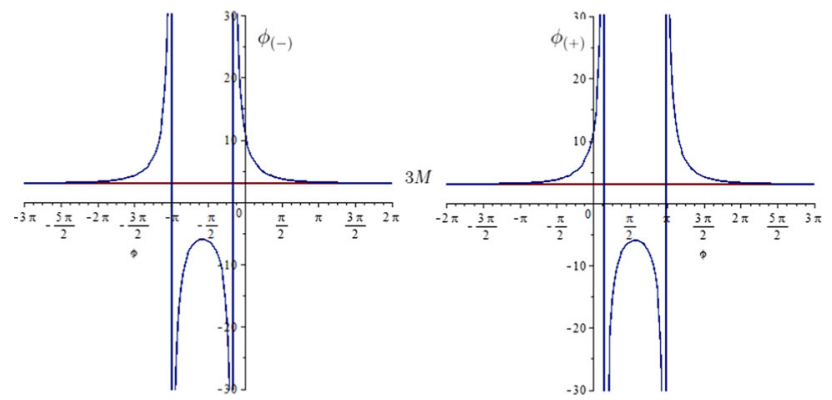

Fig. 1 The functions $r_{( \pm)}(\phi)$ of the spiral photon geodesics closest to the photon sphere of radius $3 M$

$\frac{\mathrm{d} r(\phi)}{\mathrm{d} \phi}=\frac{\mathrm{d}^{2} r(\phi)}{\mathrm{d} \phi^{2}}=0$,

giving the radius of the photon sphere $r_{\mathrm{ph}}=3 \mathrm{M}$ and the mandatory condition

$L_{p h}= \pm \frac{3 \sqrt{3} M E_{p h}}{\sqrt{1-27 \omega_{\mathrm{H}}^{2} M^{2}}}$,

derived in Ref. [15]. Thus the photons with circular geodesics are trapped on the photon sphere without escaping outside. Furthermore, by substituting the condition (14) in Eq. (12) we obtain the equation

$27 M^{2}\left(\frac{\mathrm{d} r(\phi)}{\mathrm{d} \phi}\right)^{2}-54 M^{3} r(\phi)+27 M^{2} r(\phi)^{2}-r(\phi)^{4}=0$,

which is independent on the Hubble de Sitter constant $\omega_{\mathrm{H}}$. Apart from the circular geodesics, this equation allows the solutions

$r_{( \pm)}(\phi)=3 M \frac{\left(6 M e^{\mp \phi}+1\right)^{2}}{\left(6 M e^{\mp \phi}+1\right)^{2}-36 M e^{\mp \phi}}$,

known as the spiral geodesics [47]. These are determined up to a rotation, $\phi \rightarrow \phi-\phi_{0}$, fixing the origin of this angular coordinate. For example, if we translate the arguments of the functions $r_{( \pm)}$as $\phi \rightarrow \phi^{ \pm}=\phi \pm \ln 6 M$ then we recover the elegant Darwin form [47]

$\frac{1}{r_{( \pm)}\left(\phi^{ \pm}\right)}=-\frac{1}{6 M}+\frac{1}{2 M} \tanh ^{2}\left(\frac{\phi^{ \pm}}{2}\right)$

of the spiral geodesics. Thus we may conclude that the presence of de Sitter gravity is encapsulated only in Eq. (14), while the photon sphere and the shapes of the spiral geodesics remain the same as in Minkowski flat space-time (when $\left.\omega_{\mathrm{H}}=0\right)$.
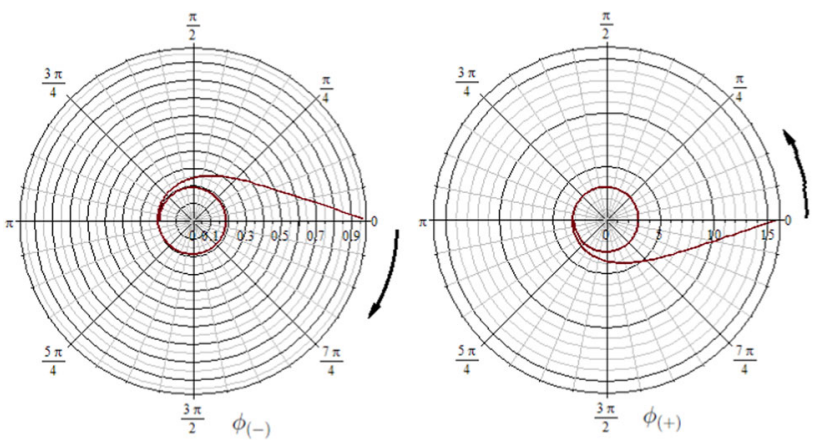

Fig. 2 The functions $r_{( \pm)}(\phi)$ of the spiral photon geodesics rolling out and escaping from the photon sphere of radius $3 M$

The spiral geodesics are symmetric, $r_{( \pm)}(\phi)=r_{(\mp)}(-\phi)$, having vertical asymptotes at

$\phi_{(+) 1,2}=-\ln \frac{2 \mp \sqrt{3}}{6 M}, \quad \phi_{(-) 1,2}=\ln \frac{2 \mp \sqrt{3}}{6 M}$.

In Fig. 1 we see that the functions $r_{( \pm)}(\phi)$ are defined on the domains $\left(-\infty, \phi_{( \pm) 1}\right) \cup\left(\phi_{( \pm) 2}, \infty\right)$, since between the vertical asymptotes their values are negative having no physical meaning. It is interesting that this opaque window is independent on the black hole mass,

$\left|\phi_{( \pm) 2}-\phi_{( \pm) 1}\right|=\ln \frac{2+\sqrt{3}}{2-\sqrt{3}} \simeq 0.8384 \pi$.

On the physical domain these trajectories remain outside the photon sphere, $r_{( \pm)}(\phi)>3 M$, but approaching to this for large $|\phi|$ since

$\lim _{|\phi| \rightarrow \infty} r_{( \pm)}(\phi)=3 M$

This gives us the image of the spiral geodesics rolled out around the photon sphere (as in Fig. 2) escaping outside only when $\phi$ is approaching to the values (18) where the functions $r_{( \pm)}$can take larger values near singularities.

The geodesics $r_{( \pm)}(\phi)$ are the closest trajectories to the photon sphere of the first photons that can be observed at the limit of the black hole shadow. Therefore, for studying this shadow and the associated redshift we have to consider only these photons.

\section{4 de Sitter isometries}

The de Sitter co-moving frames play the role of inertial frames being related among themselves through de Sitter isometries as in our de Sitter relativity $[9,10]$. Moreover, the black hole frames have the asymptotic de Sitter symmetry which governs the relative motion of the black hole 
with respect to remote observers such that we may use these isometries for relating the observer co-moving frames to the black hole one.

The de Sitter isometries can be studied easily since this manifold is a hyperboloid of radius $1 / \omega_{\mathrm{H}}$ embedded in the five-dimensional flat space-time $\left(M^{5}, \eta^{5}\right)$ of coordinates $z^{A}$ (labelled by the indices $A, B, \ldots=0,1,2,3,4$ ) and metric $\eta^{5}=\operatorname{diag}(1,-1,-1,-1,-1)$. The local charts can be introduced giving the set of functions $z^{A}(x)$ which solve the hyperboloid equation,

$\eta_{A B}^{5} z^{A}(x) z^{B}(x)=-\frac{1}{\omega_{\mathrm{H}}^{2}}$,

giving the line element

$\mathrm{d} s^{2}=\eta_{A B}^{5} \mathrm{~d} z^{A}(x) \mathrm{d} z^{B}(x)=g_{\mu \nu} \mathrm{d} x^{\mu} \mathrm{d} x^{\nu}$.

The functions that introduce our Painlevé coordinates are

$z^{0}(x)=\frac{1}{2 \omega_{\mathrm{H}}}\left[e^{\omega_{\mathrm{H}} t}-e^{-\omega_{\mathrm{H}} t}\left(1-\omega_{\mathrm{H}}^{2} \mathbf{x}^{2}\right)\right]$,

$z^{i}(x)=x^{i}$

$z^{4}(x)=\frac{1}{2 \omega_{\mathrm{H}}}\left[e^{\omega_{\mathrm{H}} t}+e^{-\omega_{\mathrm{H}} t}\left(1-\omega_{\mathrm{H}}^{2} \mathbf{x}^{2}\right)\right]$.

The de Sitter isometry group is just the stable group $\mathrm{SO}(1,4)$ of the embedding manifold $\left(M^{5}, \eta^{5}\right)$ that leave invariant its metric and implicitly Eq. (21). Therefore, given a system of coordinates defined by the functions $z=z(x)$, each transformation $\mathfrak{g} \in \mathrm{SO}(1,4)$ gives rise to the isometry $x^{\prime} \rightarrow x=\phi_{\mathfrak{g}}\left(x^{\prime}\right)$ derived from the system

$z\left[\phi_{\mathfrak{g}}\left(x^{\prime}\right)\right]=\mathfrak{g} z\left(x^{\prime}\right)$.

The local charts related through these isometries play the same role as the inertial frames of special relativity.

The classical conserved quantities under de Sitter isometries are given by the Killing vectors $k_{(A B)}$ of the de Sitter manifold [49] that are related to those of $\left(M^{5}, \eta^{5}\right)$ as

$K_{C}^{(A B)} \mathrm{d} z^{C}=z^{A} \mathrm{~d} z^{B}-z^{B} \mathrm{~d} z^{A}=k_{\mu}^{(A B)} \mathrm{d} x^{\mu}$,

allowing us to derive the covariant components of the Killing vectors in an arbitrary chart $\{x\}$ of the de Sitter space-time as

$k_{(A B) \mu}=\eta_{A C}^{5} \eta_{B D}^{5} k_{\mu}^{(C D)}=z_{A} \partial_{\mu} z_{B}-z_{B} \partial_{\mu} z_{A}$,

where $z_{A}=\eta_{A B} z^{B}$. The conserved quantities along the timelike geodesic of a particle of mass $m$ have the general form $\mathcal{K}_{(A B)}(x, \mathbf{P})=\omega_{\mathrm{H}} k_{(A B) \mu} \dot{x}^{\mu}$. The conserved quantities with physical meaning are the energy $E$, momentum $\mathbf{P}$, angular momentum $\mathbf{L}$ and a specific vector $\mathbf{Q}$ that we call the adjoint momentum [49]. A geodesic in the co-moving frame $\{t, \mathbf{x}\}$ [59],

$$
\begin{aligned}
\mathbf{x}(t)= & \mathbf{x}_{0} e^{\omega_{\mathrm{H}}\left(t-t_{0}\right)}+\frac{\mathbf{P}}{P^{2}}\left(\sqrt{m^{2}+P^{2}} e^{\omega_{\mathrm{H}}\left(t-t_{0}\right)}\right. \\
& \left.-\sqrt{m^{2} e^{2 \omega_{\mathrm{H}}\left(t-t_{0}\right)}+P^{2}}\right)
\end{aligned}
$$

depends only on the momentum $\mathbf{P}(P=|\mathbf{P}|)$ and the initial condition $\mathbf{x}\left(t_{0}\right)=\mathbf{x}_{0}$ fixed at the time $t_{0}$. The conserved quantities in an arbitrary point $(t, \mathbf{x}(t))$ of this geodesic read $[9,59]$

$$
\begin{aligned}
& E=\omega_{\mathrm{H}} \mathbf{x}(t) \cdot \mathbf{P} e^{-\omega_{\mathrm{H}}\left(t-t_{0}\right)}+\sqrt{m^{2}+P^{2} e^{-2 \omega_{\mathrm{H}}\left(t-t_{0}\right)}} \\
& \mathbf{L}=\mathbf{x}(t) \wedge \mathbf{P} \\
& \mathbf{Q}=2 \omega_{\mathrm{H}} \mathbf{x}(t) E e^{-\omega_{\mathrm{H}}\left(t-t_{0}\right)}+\mathbf{P} e^{-2 \omega_{\mathrm{H}}\left(t-t_{0}\right)}\left[1-\omega_{\mathrm{H}}^{2} \mathbf{x}(t)^{2}\right]
\end{aligned}
$$

satisfying the obvious identity

$E^{2}-\omega_{\mathrm{H}}^{2} \mathbf{L}^{2}-\mathbf{P} \cdot \mathbf{Q}=m^{2}$,

corresponding to the first Casimir invariant of the $\operatorname{SO}(1,4)$ algebra [49]. In the flat limit, when $\omega_{\mathrm{H}} \rightarrow 0$, we have $\mathbf{Q} \rightarrow$ $\mathbf{P}$ such that this identity becomes just the usual mass-shell condition, $E^{2}-\mathbf{P}^{2}=m^{2}$, of special relativity.

The conserved quantities $E, \mathbf{P}$ and the new ones,

$\mathbf{K}=-\frac{1}{2 \omega_{\mathrm{H}}}(\mathbf{P}-\mathbf{Q}), \quad \mathbf{R}=-\frac{1}{2 \omega_{\mathrm{H}}}(\mathbf{P}+\mathbf{Q})$,

form a skew-symmetric tensor on $M^{5}$,

$\mathcal{K}(x, \mathbf{P})=\left(\begin{array}{ccccc}0 & \omega_{\mathrm{H}} K_{1} & \omega_{\mathrm{H}} K_{2} & \omega_{\mathrm{H}} K_{3} & E \\ -\omega_{\mathrm{H}} K_{1} & 0 & \omega_{\mathrm{H}} L_{3} & -\omega_{\mathrm{H}} L_{2} & \omega_{\mathrm{H}} R_{1} \\ -\omega_{\mathrm{H}} K_{2} & -\omega_{\mathrm{H}} L_{3} & 0 & \omega_{\mathrm{H}} L_{1} & \omega_{\mathrm{H}} R_{2} \\ -\omega_{\mathrm{H}} K_{3} & \omega_{\mathrm{H}} L_{2} & -\omega_{\mathrm{H}} L_{1} & 0 & \omega_{\mathrm{H}} R_{3} \\ -E & -\omega_{\mathrm{H}} R_{1} & -\omega_{\mathrm{H}} R_{2} & -\omega_{\mathrm{H}} R_{3} & 0\end{array}\right)$,

whose components transform under the isometries $x^{\prime} \rightarrow x=$ $\phi_{\mathfrak{g}}\left(x^{\prime}\right)$ defined by Eq. (24) as

$\mathcal{K}(t, \mathbf{x}, \mathbf{P})=\overline{\mathfrak{g}} \mathcal{K}^{\prime}\left(t^{\prime}, \mathbf{x}^{\prime}, \mathbf{P}^{\prime}\right) \overline{\mathfrak{g}}^{T}$,

where $\overline{\mathfrak{g}}=\eta^{5} \mathfrak{g} \eta^{5}$ [9].

Summarizing, we can say that the de Sitter isometries are generated globally by the $S O(1,4)$ transformations which determine the transformations of the coordinates and conserved quantities. We have thus a specific relativity on the de Sitter space-time allowing us to study different relativistic effects in the presence of the de Sitter gravity. In what follows we use the Lorentzian isometries defined in Ref. [9] and the translations presented in the Appendix A. 


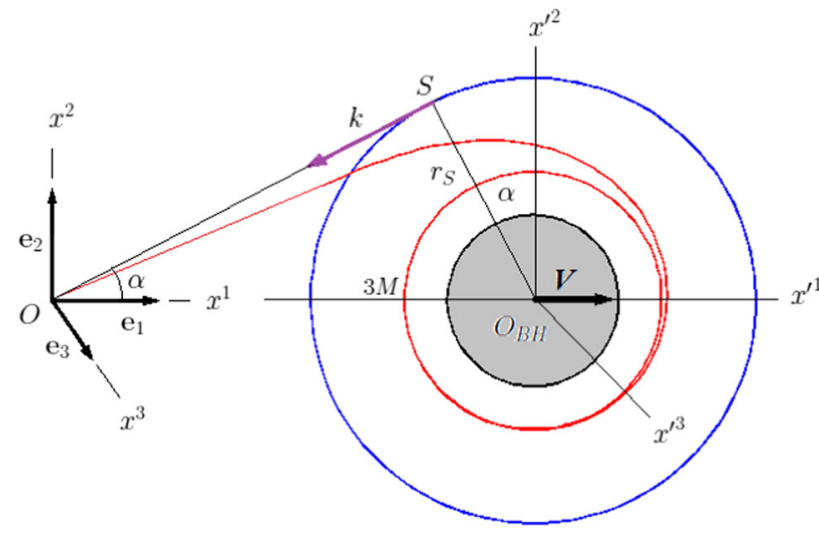

Fig. 3 The geodesic $r_{(+)}(\phi)$ observed as a rectilinear de Sitter one of a photon of momentum $k$ emitted by the apparent source $S$ situated on the sphere of radius $r_{S}>3 \sqrt{3} M$

\section{Observing light from black holes}

Le us consider now a mobile black hole in its proper comoving frame $\left\{t^{\prime}, \mathbf{x}^{\prime}\right\}_{B H}$ with the origin in $O_{B H}$ and a fixed remote observer in his own co-moving frame $\{t, \mathbf{x}\}$ having the origin in $O$. We consider that the space Cartesian axes of these frames remain parallel with the basis of unit vectors $\left(\mathbf{e}_{1}, \mathbf{e}_{2}, \mathbf{e}_{3}\right)$ such that the geodesic of the emitted photon is in the plane $\left(\mathbf{e}_{1}, \mathbf{e}_{2}\right)$. In this geometry we assume that the photon is emitted at the initial moment $t=t^{\prime}=0$ when the origin $O_{B H}$ is translated with $d$ and has the relative velocity $\mathbf{V}=\mathbf{e}_{1} V$ with respect to $O$. Note that the velocity $\mathbf{V}=\frac{\mathbf{P}}{M}$ is conserved depending on the conserved momentum $\mathbf{P}$ of the black hole geodesic observed by $O$.

\subsection{Related conserved quantities}

A remote observer sees the photon of momentum $\mathbf{k}=\mathbf{n}_{k} k$, energy $E_{p h}=|\mathbf{k}|$ and angular momentum (14), as emitted from an apparent source $S$ of position vector $\mathbf{n}_{S} r_{S}$ on the sphere of radius

$r_{S}=\frac{|L|}{k}=\frac{3 \sqrt{3} M}{\sqrt{1-27 \omega_{\mathrm{H}}^{2} M^{2}}}$

which is just the apparent radius of the black hole shadow. Therefore, $r_{S}$ is the radius of the sphere hosting different photon sources $S$ that can be observed nearest to the black hole shadow (as in Fig. 3). When $\omega_{\mathrm{H}} \rightarrow 0$ this becomes just the shadow radius $3 \sqrt{3} M$ derived in special relativity [47].

The apparent trajectory of the emitted photon is a de Sitter null geodesic of momentum $\mathbf{k}$ that, according to Eq. (27), reads

$\mathbf{x}_{p h}(t)=\mathbf{n}_{S} r_{S} e^{\omega_{\mathrm{H}} t}+\mathbf{n}_{k}\left(e^{\omega_{\mathrm{H}} t}-1\right)$ complying with the initial condition $\mathbf{x}_{p h}(0)=\mathbf{n}_{S} r_{S}$. This geodesic depends on the orthogonal unit vectors, which can be represented as

$\mathbf{n}_{k}=-\mathbf{e}_{1} \cos \alpha-\mathbf{e}_{2} \sin \alpha$,

$\mathbf{n}_{S}=-\mathbf{e}_{1} \sin \alpha+\mathbf{e}_{2} \cos \alpha$,

where the angle $\alpha$, giving the apparent direction of the photon, will depend on observer's position. We have thus the opportunity of defining the de Sitter conserved quantities on this geodesic as in an apparent de Sitter empty comoving frame $\left\{t^{\prime}, \mathbf{x}^{\prime}\right\}$ associated to $\left\{t^{\prime}, \mathbf{x}^{\prime}\right\}_{B H}$. Here one vector is missing, namely the adjoint momentum $\mathbf{Q}^{\prime}$ that can be derived simply at the time $t=0$ according to Eq. (30). We complete thus the set of conserved quantities,

$E^{\prime}=k$,

$\mathbf{P}^{\prime}=\mathbf{n}_{k} k$,

$\mathbf{L}^{\prime}=\mathbf{e}_{3} r_{S} k$,

$\mathbf{Q}^{\prime}=\mathbf{n}_{S} 2 \omega_{\mathrm{H}} r_{S} k+\mathbf{n}_{k} k\left(1-\omega_{\mathrm{H}}^{2} r_{S}^{2}\right)$,

which satisfy the condition (31).

Now we can deduce how these conserved quantities are measured by the fixed observer $O$ since the observer frame $\{t, \mathbf{x}\}$ and the apparent black hole one, $\left\{t^{\prime}, \mathbf{x}^{\prime}\right\}$, are related through an isometry, $x=\phi_{\mathfrak{g}}\left(x^{\prime}\right)$, of the de Sitter relativity [9]. According to our hypotheses, this is generated by the $\mathrm{SO}(1,4)$ transformation,

$\mathfrak{g}=\mathfrak{g}(\mathbf{V}) \mathfrak{g}(\mathbf{a})$,

formed by a translation (A.1) of parameter $\mathbf{a}=\mathbf{e}_{1} d=$ $(d, 0,0)$, having the form $[9,60]$

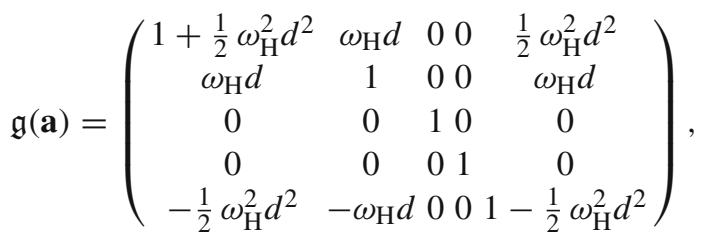

followed by the Lorentz boost

$\mathbf{V}=(V, 0,0) \rightarrow \mathfrak{g}(\mathbf{V})=\left(\begin{array}{ccccc}\frac{1}{\sqrt{1-V^{2}}} & \frac{V}{\sqrt{1-V^{2}}} & 0 & 0 & 0 \\ \frac{V}{\sqrt{1-V^{2}}} & \frac{1}{\sqrt{1-V^{2}}} & 0 & 0 & 0 \\ 0 & 0 & 1 & 0 & 0 \\ 0 & 0 & 0 & 1 & 0 \\ 0 & 0 & 0 & 0 & 1\end{array}\right)$

of the particular Lorentzian isometry we need here [9].

Applying then the transformation (34) with $\mathfrak{g}$ given by Eq. (43) we obtain the conserved quantities observed by $O$. This calculation is elementary but complicated, involving many terms that can be manipulated only by using suitable 
algebraic codes on computer. For presenting the final result it is convenient to introduce the notation

$\delta=\omega_{\mathrm{H}} d=\frac{d}{l_{\mathrm{H}}}, \quad \xi=\frac{r_{S}}{d}$

which allows us to write down the conserved quantities in the observer's frame as

$$
\begin{aligned}
& E=\frac{k}{\sqrt{1-V^{2}}}[1-V \cos \alpha+\delta(V-\cos \alpha-\xi V \sin \alpha) \\
& \left.-\frac{\delta^{2}}{2}\left(1-\xi^{2}\right) V \cos \alpha\right] \text {, } \\
& P^{1}=k\left(\frac{1}{\sqrt{1-V^{2}}}-1\right) \\
& \times\left[\delta(1-\xi \sin \alpha)-\frac{\delta^{2}}{2}\left(1-\xi^{2}\right) \cos \alpha\right] \\
& +\frac{k}{\sqrt{1-V^{2}}}[V(1-\delta \cos \alpha)-\cos \alpha], \\
& P^{2}=k\left(\frac{1}{\sqrt{1-V^{2}}}-1\right) \\
& \times\left[\delta \xi(\delta-\cos \alpha)-\frac{\delta^{2}}{2}\left(1+\xi^{2}\right) \sin \alpha\right] \\
& -k \sin \alpha+\frac{k \delta V}{\sqrt{1-V^{2}}}(\xi-\sin \alpha), \\
& Q^{1}=k\left(\frac{1}{\sqrt{1-V^{2}}}+1\right) \\
& \times\left[\delta(1-\xi \sin \alpha)-\frac{\delta^{2}}{2}\left(1-\xi^{2}\right) \cos \alpha\right] \\
& +\frac{k}{\sqrt{1-V^{2}}}[V(1-\delta \cos \alpha)-\cos \alpha] \text {, } \\
& Q^{2}=k\left(\frac{1}{\sqrt{1-V^{2}}}+1\right) \\
& \times\left[-\delta \xi(\delta-\cos \alpha)+\frac{\delta^{2}}{2}\left(1+\xi^{2}\right) \sin \alpha\right] \\
& -k \sin \alpha-\frac{k \delta V}{\sqrt{1-V^{2}}}(\xi-\sin \alpha), \\
& L_{3}=\frac{k \delta}{\omega_{\mathrm{H}} \sqrt{1-V^{2}}}[\xi(1-V \cos \alpha)-\sin \alpha+\delta \xi V \\
& \left.-\frac{\delta V}{2}\left(1+\xi^{2}\right) \sin \alpha\right] \text {, }
\end{aligned}
$$

while $L_{1}=L_{2}=P^{3}=Q^{3}=0$. As expected, these quantities satisfy the invariant identity (31).

In other respects, we observe that all the vector components we derived above can take any real values in contrast with the energy which must remain positive definite. This condition is fulfilled only if
$V<V_{\lim }=\frac{2(1-\delta \cos \alpha)}{\delta^{2}\left(1-\xi^{2}\right) \cos \alpha-2 \delta(1-\xi \sin \alpha)+2 \cos \alpha}$

This is in fact the mandatory condition for observing the photon in $O$ at finite time. When the relative velocity $V$ exceeds this limit then the photon cannot arrive in $O$ at finite time because of the background expansion. Thus $V_{\text {lim }}$ defines a new velocity horizon restricting the velocities such that for very far sources with $\alpha=0$ and $\delta=\omega_{\mathrm{H}} d \sim 1$ this limit vanishes.

\subsection{Shadow and redshift}

The angle $\alpha$, which depends on the relative position between black hole and observer, can be found simply imposing the condition $L_{3}=0$ when the photon is passing through the point $O$. Solving this equation for $L_{3}$ given by Eq. (52) we find

$$
\tan \frac{\alpha}{2}=\frac{\delta V\left(1+\xi^{2}\right)+2-\sqrt{\left[V \delta\left(1-\xi^{2}\right)+2\right]^{2}+4 \xi^{2}\left(V^{2}-1\right)}}{2 \xi(V \delta+V+1)} .
$$

Substituting then this angle in Eqs. (47)-(53) we obtain all the conserved quantities measured by $O$ and the maximal velocity $V_{\text {lim. }}$.

Hereby we can extract the quantities of general interest, namely $\sin \alpha$, measured in the black hole proper frame, the angular radius

$\sin \alpha_{\mathrm{obs}}=\frac{\left|P^{2}\right|}{P}, \quad P=\sqrt{P^{1^{2}+P^{2}}}$

of the shadow, measured in the observer's frame, and the redshift $z$ defined as

$1+z=\frac{E^{\prime}}{E}=\frac{k}{E}$

Unfortunately, the exact expressions of these quantities have a huge number of terms that cannot be written here but can be manipulated on computer [1] for extracting significant particular cases or intuitive approximations.

The simplest particular case is when the black hole does not have a initial relative velocity with respect to $O$. Then by setting $V=0$ we obtain the simple formulas

$$
\begin{aligned}
\sin \alpha_{\mathrm{obs}} & =\sin \alpha=\xi=\frac{r_{S}}{d}, \\
\frac{1}{1+z} & =1-\omega_{\mathrm{H}} d \cos \alpha=1-\omega_{\mathrm{H}} d \sqrt{1-\xi^{2}},
\end{aligned}
$$

showing how the observations of the shadow and redshift are related each other. 
In the general case of $V \neq 0$, we observe that the expansions around $\xi=0$ are useful since this is the only parameter which remains very small in astronomical observations as long as the other ones have larger ranges, $0<\delta=\omega_{\mathrm{H}} d<1$ and $0<V<V_{\text {lim }}$. Computing these series we write down here only the lowest terms

$$
\begin{aligned}
\sin \alpha & =\frac{2\left(1+\omega_{\mathrm{H}} \mathrm{d} V-V\right)}{2+\omega_{\mathrm{H}} \mathrm{d} V} \xi+\mathcal{O}\left(\xi^{3}\right) \\
\sin \alpha_{\mathrm{obs}} & =\frac{2 \sqrt{1-V^{2}}}{2+\omega_{\mathrm{H}} \mathrm{d} V\left(1-\xi^{2}\right)} \xi \\
& =\frac{2 \sqrt{1-V^{2}}}{2+\omega_{\mathrm{H}} \mathrm{d} V} \xi+\mathcal{O}\left(\xi^{3}\right) \\
\frac{1}{1+z} & =\sqrt{\frac{1-V}{1+V}}\left(1-\omega_{\mathrm{H}} d-\frac{\omega_{\mathrm{H}}^{2} d^{2}}{2} \frac{V}{1-V}\right)+\mathcal{O}\left(\xi^{2}\right)
\end{aligned}
$$

which are still comprehensible and can be interpreted. Similarly, for the velocity limit we have

$V_{\lim }=\frac{2\left(1-\omega_{\mathrm{H}} d\right)}{1+\left(1-\omega_{\mathrm{H}} d\right)^{2}}+\mathcal{O}\left(\xi^{2}\right)$.

The principal novelty here is that $\sin \alpha_{\text {obs }}$ depends on the relative velocity $V$ as in Eq. (60) just from the first order of the expansion which may be observationally accessible. In contrast, the expansion (61) has a first term independent on $\xi$ recovering just the redshift formula we derived recently for a point-like source moving along the $\mathbf{e}_{1}$ axis [11]. Therefore, the influence of the black hole dimensions could be observed only when the second term of the order $\mathcal{O}\left(\xi^{2}\right)$ could be measured with a satisfactory accuracy.

\subsection{Flat limit}

The limit of the de Sitter relativity when the de Sitter-Hubble constant $\omega_{\mathrm{H}}$ vanishes is just the usual version of special relativity. Then all the co-moving frames of the de Sitter relativity become inertial frames in Minkowski space-time without affecting the black hole geometry in its proper frame. Now a remote observer sees a photon emitted in $S$ as having an apparent rectilinear trajectory with momentum $k$ and energy $E_{p h}=k$. Then all the measured quantities can be obtained from the de Sitter ones in the limit $\omega_{\mathrm{H}} \rightarrow 0$. In this limit our principal parameters becomes

$r_{S} \rightarrow \hat{r}_{S}=3 \sqrt{3} M, \quad \xi \rightarrow \hat{\xi}=\frac{3 \sqrt{3} M}{d}$,

while the other quantities have the limits

$$
\sin \hat{\alpha}=\lim _{\omega_{\mathrm{H}} \rightarrow 0} \sin \alpha=(1-V) \hat{\xi} \Delta,
$$

$$
\begin{aligned}
\sin \hat{\alpha}_{\mathrm{obs}} & =\lim _{\omega_{\mathrm{H}} \rightarrow 0} \sin \alpha_{\mathrm{obs}}=\sqrt{1-V^{2}} \hat{\xi}, \\
\frac{1}{1+\hat{z}} & =\lim _{\omega_{\mathrm{H}} \rightarrow 0} \frac{1}{1+z}=\sqrt{\frac{1-V}{1+V}} \Delta,
\end{aligned}
$$

where

$$
\begin{aligned}
\Delta & =\frac{1+V}{1+V \cos \hat{\alpha}_{\text {obs }}}=\frac{1+V}{1+V \sqrt{1-\hat{\xi}^{2}\left(1-V^{2}\right)}} \\
& =1+V(1-V) \frac{\hat{\xi}^{2}}{2}+\mathcal{O}\left(\hat{\xi}^{4}\right) .
\end{aligned}
$$

As expected, hereby we recover the usual aberration formulas

$\sin \hat{\alpha}=\frac{\sqrt{1-V^{2}} \sin \hat{\alpha}_{\mathrm{obs}}}{1+V \cos \hat{\alpha}_{\mathrm{obs}}} \Leftrightarrow \sin \hat{\alpha}_{\mathrm{obs}}=\frac{\sqrt{1-V^{2}} \sin \hat{\alpha}}{1-V \cos \hat{\alpha}}$.

In addition, we obtain the limit velocity

$\hat{V}_{\lim }=\lim _{\omega_{\mathrm{H}} \rightarrow 0} V_{\lim }=\frac{1}{\cos \hat{\alpha}} \geq 1$,

which does not make sense since this can exceed the speed of light. Thus the limitation of velocities disappears together with the de Sitter event horizon.

For interpreting these results a good choice is $\Delta \simeq 1$ as the parameter $\xi$ remains very small. Then Eq. (66) is just the redshift due to the Doppler effect in special relativity. Moreover, we may convince ourselves that all the limits derived above are just the results that may be obtained by applying our method in special relativity, as presented briefly in Appendix B.

\section{Concluding remarks}

We studied how a co-moving observer measures simultaneously the shadow and redshift of a Scwarzschild black hole freely falling in the de Sitter expanding universe. For this purpose we used a new algebraic method offered by our de Sitter relativity that provides us with suitable isometries transforming the conserved quantities of the emitted photon into those recorded by a remote co-moving observer. In this manner we obtained the closed formula (60) of the shadow depending on the peculiar velocity and the corrections to the new redshift formula (61) that can be calculated on computer by using the code [1].

Another advantage of our method is that this is somewhat independent on the coordinates which are involved only in imposing the initial conditions. For example, the choice of the co-moving frames with Painlevé coordinates simplifies the calculations since then we use the translation (2) which 
does not affect the time. In contrast, in static coordinates, defined by Eq. (4), the same translation gives the transformation (6) which affects the time such that it is more difficult to synchronise the clocks by setting common initial conditions when $V \neq 0$. However, this is not a real impediment as long as we know how the coordinates transform among themselves.

This relative independence on coordinates can be tested in the case of $V=0$ applying our method to a static black hole. Our preliminary calculations indicate that the shadow of the static black hole is given by Eq. (57) just as in the case of the co-moving frames with Painleve coordinates. This stability comes from the fact that in both cases we start with the same conserved quantities (39)-(42) transformed by the same translation (44) whose parameter $d$ is the physical distance between black hole and observer.

Under such circumstances we may compare how the algebraic and geometric methods work in determining the black hole shadow at least in the case of $V=0$. The shadow formula derived in Ref. [15] by using the geometric method can be written in our notation as $\sin \alpha_{\mathrm{obs}}=\frac{r_{S}}{d^{\prime}} \sqrt{f\left(d^{\prime}\right)}$ where $d^{\prime}$ is now the radial coordinate of the fixed observer. Thus we see that in our approach the factor $\sqrt{f\left(d^{\prime}\right)}$ is missing. This means that between these two methods there are some minor differences that may come from the approximation of remote observers on which the algebraic method is based and from the fact that in the static frame the radial coordinate $d^{\prime}$ does not coincide with the physical distance $d$. However, now it is premature to say more about this relationship before analyzing many examples in de Sitter relativity.

In other respects, we must specify that the study of the conserved quantities is not enough for understanding the entire information carried out by the light emitted by moving black holes. There are important observable quantities resulted from the coordinate transformations under isometries as, for example, the photon propagation time or the real distance between observer and black hole at the time when the photon is measured. In Ref. [11] we derived such quantities in the longitudinal case of a point-like source moving along the observer-black hole direction. Therefore, when we apply the algebraic method the coordinate transformations under isometries or other geometric tools may complete our investigation.

We hope that the algebraic method proposed here will improve the general geometric approach for getting over the difficulties in analyzing the light emitted by various cosmic objects moving in the de Sitter expanding universe.

Data Availability Statement This manuscript has no associated data or the data will not be deposited. [Authors' comment: There are no experimental or numerical data. The code [1] is exclusively algebraic.]

Open Access This article is licensed under a Creative Commons Attribution 4.0 International License, which permits use, sharing, adaptation, distribution and reproduction in any medium or format, as long as you give appropriate credit to the original author(s) and the source, provide a link to the Creative Commons licence, and indicate if changes were made. The images or other third party material in this article are included in the article's Creative Commons licence, unless indicated otherwise in a credit line to the material. If material is not included in the article's Creative Commons licence and your intended use is not permitted by statutory regulation or exceeds the permitted use, you will need to obtain permission directly from the copyright holder. To view a copy of this licence, visit http://creativecomm ons.org/licenses/by/4.0/.

Funded by $\mathrm{SCOAP}^{3}$.

\section{Appendix A: Translations}

The space translations of the $S O(1,4)$ group are less used in applications such that it is worth reviewing briefly their action in different coordinates. A space translation of parameters $\mathbf{a}=\left(a^{1}, a^{2}, a^{3}\right)$ is the isometry $x^{\prime} \rightarrow x=\phi_{\mathfrak{g}(\mathbf{a})}\left(x^{\prime}\right)$ generated by the $\mathrm{SO}(1,4)$ transformation $[9,60]$,

$\mathfrak{g}(\mathbf{a})=\left(\begin{array}{ccccc}1+\frac{1}{2} \omega_{\mathrm{H}}^{2} \mathbf{a}^{2} & \omega_{\mathrm{H}} a^{1} & \omega_{\mathrm{H}} a^{2} & \omega_{\mathrm{H}} a^{3} & \frac{1}{2} \omega_{\mathrm{H}}^{2} \mathbf{a}^{2} \\ \omega_{\mathrm{H}} a^{1} & 1 & 0 & 0 & \omega_{\mathrm{H}} a^{1} \\ \omega_{\mathrm{H}} a^{2} & 0 & 1 & 0 & \omega_{\mathrm{H}} a^{2} \\ \omega_{\mathrm{H}} a^{3} & 0 & 0 & 1 & \omega_{\mathrm{H}} a^{3} \\ -\frac{1}{2} \omega_{\mathrm{H}}^{2} \mathbf{a}^{2} & -\omega_{\mathrm{H}} a^{1} & -\omega_{\mathrm{H}} a^{2} & -\omega_{\mathrm{H}} a^{3} & 1-\frac{1}{2} \omega_{\mathrm{H}}^{2} \mathbf{a}^{2}\end{array}\right)$

according to Eq. (24) where now $\mathfrak{g}=\mathfrak{g}(\mathbf{a})$. Solving this equation in the co-moving frames with Painlevé coordinates where the $z$-functions have the form (23) we find the transformation

$$
\begin{aligned}
& t=t^{\prime} \\
& \mathbf{x}=\mathbf{x}^{\prime}+\mathbf{a} e^{\omega_{\mathrm{H}} t},
\end{aligned}
$$

which does not affect the time but is not static. The only genuine static translations transform the conformal coordinates

$$
\begin{aligned}
& t_{c}=-\frac{1}{\omega_{\mathrm{H}}} e^{-\omega_{\mathrm{H}} t}, \\
& \mathbf{x}_{c}=\mathbf{x} e^{-\omega_{\mathrm{H}} t},
\end{aligned} \rightarrow \begin{aligned}
& t_{c}=t_{c}^{\prime}, \\
& \mathbf{x}_{c}=\mathbf{x}_{c}^{\prime}+\mathbf{a} .
\end{aligned}
$$

The the static coordinates $\left(t_{s}, \mathbf{x}\right)$ where

$t_{s}=t-\frac{1}{\omega_{\mathrm{H}}} \ln \chi(r), \quad \chi(r)=\sqrt{1-\omega_{\mathrm{H}}^{2} r^{2}}$,

with $r=|\mathbf{x}|$, as defined by Eq. (5), can be introduced by the functions

$$
\begin{aligned}
z^{0}\left(t_{s}, \mathbf{x}\right) & =\frac{1}{\omega_{\mathrm{H}}} \quad \chi(r) \sinh \omega_{\mathrm{H}} t_{s}, \\
z^{i}\left(t_{s}, \mathbf{x}\right) & =x^{i}, \\
z^{4}\left(t_{s}, \mathbf{x}\right) & =\frac{1}{\omega_{\mathrm{H}}} \quad \chi(r) \cosh \omega_{\mathrm{H}} t_{s} .
\end{aligned}
$$


Solving again Eq. (24) for these functions and the transformation (A.1) we find the transformation rules under translations,

$t_{s}=\frac{1}{2 \omega_{\mathrm{H}}} \ln \left(\frac{\chi\left(r^{\prime}\right)}{\chi\left(r^{\prime}\right) e^{-2 \omega_{\mathrm{H}} t_{s}^{\prime}}-2 \omega_{\mathrm{H}}^{2} \mathbf{a} \cdot \mathbf{x}^{\prime} e^{-\omega_{\mathrm{H}} t_{s}^{\prime}}-\omega_{\mathrm{H}}^{2} \mathbf{a}^{2} \chi\left(r^{\prime}\right)}\right)$,

$\mathbf{x}=\mathbf{x}^{\prime}+\mathbf{a} \chi\left(r^{\prime}\right) e^{\omega_{\mathrm{H}} t_{s}^{\prime}}$

which we present here for the first time.

\section{Appendix B: Moving black holes in special relativity}

Let us consider now a Schwarzschild black hole embedded in the flat space-time where the remote observers stay in inertial frames. We apply our algebraic method assuming that at the initial time $t=0$ the black hole origin $O_{B H}$ is translated with $d$ and moves with the relative velocity $\mathbf{V}=(V, 0,0)$ with respect to $O$. At the same time, the apparent source $S$ of coordinates

$x^{\prime}=\left(0,-\hat{r}_{S} \sin \hat{\alpha},-\hat{r}_{S} \cos \hat{\alpha}, 0\right)^{T}$,

emits a photon of energy-momentum

$p^{\prime}=(k,-k \cos \hat{\alpha},-k \sin \hat{\alpha}, 0)^{T}$,

which has to be observed in the fixed origin $O$.

The black hole and observer frames, $O_{B H}$ and $O$, are related through the isometry $\Lambda=L(\mathbf{V}) T(d)$ formed by the translation $T(d): x^{\prime 1} \rightarrow x^{1}=x^{\prime 1}+d$ followed by a Lorentz transformation $L(\mathbf{V})$, which is just the fourdimensional restriction of the matrix (45). Performing this isometry we obtain the four-vectors $x=\Lambda x^{\prime}$ and $p=\Lambda p^{\prime}$ whose components give the energy and angular momentum observed in $O$ as

$$
\begin{aligned}
E & =p^{0}=\frac{k(1-V \cos \hat{\alpha})}{\sqrt{1-V^{2}}}, \\
L_{3} & =x^{1} p^{2}-x^{2} p^{1}=\frac{k d[\xi(1-V \cos \hat{\alpha})-\sin \hat{\alpha}]}{\sqrt{1-V^{2}}} .
\end{aligned}
$$

The condition $L_{3}=0$ of the photon passing through $O$ gives

$\tan \frac{\hat{\alpha}}{2}=\frac{1-\sqrt{1-\hat{\xi}^{2}\left(1-V^{2}\right)}}{\hat{\xi}(1+V)}$.

In observer's frame the black hole shadow is seen under the angle $\hat{\alpha}_{\text {obs }}$ defined as

$\sin \hat{\alpha}_{\text {obs }}=\frac{\left|p^{2}\right|}{p}, \quad p=\sqrt{p^{1^{2}}+p^{22}}$.
Finally, calculating $\sin \hat{\alpha}$ and $\cos \hat{\alpha}$ and substituting these values in Eqs. (B.3) and (B.6) we verify that the limits (64)(69) are correct.

\section{References}

1. I.I. Cotăescu, Maple code BH01 (2020). https://physics.uvt.ro/ $\sim \operatorname{cota} / \mathrm{CCFT} /$ codes

2. G.E. Lemaître, Ann. Soc. Sci. de Bruxelles 47A, 49 (1927)

3. G.E. Lemaître, MNRAS 91, 483 (1931)

4. E. Hubble, Proc. Natl. Acad. Sci. 15, 168 (1929)

5. S. Weinberg, Gravitation and Cosmology: Principles and Applications of the General Theory of relativity (Wiley, New York, 1972)

6. E.R. Harrison, Cosmology: The Science of the Universe (Cambridge Univ. Press, New York, 1981)

7. E. Harrison, Astrophys. J. 403, 28 (1993)

8. L.D. Landau, E.M. Lifshitz, The Classical Theory of Fields (Elsevier Sci. Inc, New York, 1975)

9. I.I. Cotăescu, Eur. Phys. J. C 77, 485 (2017)

10. I.I. Cotăescu, Eur. Phys. J. C 78, 95 (2018)

11. I.I. Cotăescu, arXiv:2007.13590 (in press at MPLA)

12. K. Akiyama et al., Astrophys. J. 875(1), L1 (2019)

13. K. Akiyama et al., Astrophys. J. 875(1), L6 (2019)

14. J.L. Synge, Mon. Not. R. Astron. Soc. 131, 463 (1966)

15. V. Perlick, OYu. Tsupko, G.S. Bisnovatyi-Kogan, Phys. Rev. D 97, 104062 (2018)

16. G.S. Bisnovatyi-Kogan, OYu. Tsupko, Phys. Rev. D 98, 084020 (2018)

17. J.T. Firouzjaee, A. Allahyari, Eur. Phys. J. C 79, 1140 (2019)

18. Z. Chang, Q.-H. Zhu, JCAP 06, 055 (2020)

19. S. Vagnozzi, C. Bambi, L. Visinelli, Class. Quantum Gravity 37, 087001 (2020)

20. OYu. Tsupko, G.S. Bisnovatyi-Kogan, Int. J. Mod. Phys. D 29, $2050062(2020)$

21. J.M. Bardeen, Proceedings, Ecole d'Et de Physique Thorique: Les Astres Occlus (Les Houches, France, 1973)

22. H. Falcke, F. Melia, E. Agol, Astrophys. J. Lett. 528, L13 (1999)

23. A. Grenzebach, V. Perlick, C. Lämmerzahl, Phys. Rev. D 89, 124004 (2014)

24. Z. Stuchlik, D. Charbulák, J. Schee, Eur. Phys. J. C 78, 180 (2018)

25. P.-C. Li, M. Guo, B. Chen, Phys. Rev. D 101, 084041 (2020)

26. Z. Chang, Q.-H. Zhu, Phys. Rev. D 101, 084029 (2020)

27. F. Atamurotov, A. Abdujabbarov, B. Ahmedov, Phys. Rev. D 88, 064004 (2013)

28. Z. Li, C. Bambi, J. Cosmol. Astropart. Phys. 01, 041 (2014)

29. A. Grenzebach, V. Perlick, C. Lämmerzahl, Int. J. Mod. Phys. D 24, 1542024 (2015)

30. P.V.P. Cunha, C.A.R. Herdeiro, E. Radu, H.F. Runarsson, Phys. Rev. Lett. 115, 211102 (2015)

31. A. Abdujabbarov, M. Amir, B. Ahmedov, S.G. Ghosh, Phys. Rev. D 93, 104004 (2016)

32. C. Bambi, K. Freese, S. Vagnozzi, L. Visinelli, Phys. Rev. D 100, 044057 (2019)

33. S. Vagnozzi, L. Visinelli, Phys. Rev. D 100, 024020 (2019)

34. K. Jusu, M. Jamil, P. Salucci, T. Zhu, S. Haroon, Phys. Rev. D 100, 044012 (2019)

35. P.V.P. Cunha, C.A.R. Herdeiro, E. Radu, Universe 5, 220 (2019)

36. S. Haroon, M. Jamil, K. Jusu, K. Lin, R.B. Mann, Phys. Rev. D 99 , 044015 (2019)

37. S.-W. Wei, Y.-X. Liu, R.B. Mann, Phys. Rev. D 99, 041303 (2019)

38. N. Bar, K. Blum, T. Lacroix, P. Panci, J. Cosmol. Astropart. Phys. 07, 045 (2019)

39. H. Davoudiasl, P.B. Denton, Phys. Rev. Lett. 123, 021102 (2019) 
40. I. Banerjee, S. Chakraborty, S. SenGupta, Phys. Rev. D 101, 041301 (2020)

41. R. Roy, U.A. Yajnik, Phys. Lett. B 803, 135284 (2020)

42. R. Roy, S. Chakrabarti, Phys. Rev. D 102, 024059 (2020)

43. C. Li, S.-F. Yan, L. Xue, X. Ren, Y.-F. Cai, D.A. Easson, Y.-F. Yuan, H. Zhao, Phys. Rev. Res. 2, 023164 (2020)

44. P. Painlevé, C. R. Acad. Sci. (Paris) 173, 677 (1921)

45. N.D. Birrel, P.C.W. Davies, Quantum Fields in Curved Space (Cambridge University Press, Cambridge, 1982)

46. F. Kottler, Ann. Phys. (Berlin) 361, 401 (1918)

47. C. Darwin, Proc. R. Soc. 249, 180 (1958)

48. C. Darwin, Proc. R. Soc. 263, 39 (1961)

49. I.I. Cotăescu, GRG 43, 1639 (2011)

50. G.W. Gibbons, C.M. Warnick, M.C. Werner, Class. Quantum Gravity 25, 245009 (2008)
51. T. Treu, R.S. Ellis, Contemp. Phys. 56(1), 17 (2015)

52. A.S. Eddington, The Mathematical Theory of Relativity (Cambridge University Press, Cambridge, 1923)

53. C.M. Claudel, K.S. Virbhadra, G.F.R. Ellis, J. Math. Phys. 42, 818 (2001)

54. K.S. Virbhadra, G.F.R. Ellis, Phys. Rev. D 62, 084003 (2000)

55. K.S. Virbhadra, G.F.R. Ellis, Phys. Rev. D 65, 103004 (2002)

56. K.S. Virbhadra, D. Narasimha, S.M. Chitre, Astron. Astrophys. 337, 1 (1998)

57. K.S. Virbhadra, C.R. Keeton, Phys. Rev. D 77, 124014 (2008)

58. K.S. Virbhadra, Phys. Rev. D 79, 083004 (2009)

59. I.I. Cotăescu, Mod. Phys. Lett. A 32, 1750223 (2017)

60. O. Nachtmann, Commun. Math. Phys. 6, 1 (1967) 